\title{
XAS and XRD analysis of active Pt and Pd sites in metal-organic framework UiO-67
}

\author{
A. Skorynina ${ }^{1}$, A. Bugaev ${ }^{1}$, K. Lomachenko ${ }^{2}$, A. Guda ${ }^{1}$, A. Lazzarini ${ }^{3}$, U. Olsbye ${ }^{3}$, K. Lillerud ${ }^{3}$, A. Soldatov ${ }^{1}$ \\ ${ }^{1}$ Southern Federal University, Rostov-on-Don, Russia, ${ }^{2}$ ESRF, Grenoble, France, ${ }^{3}$ University of Oslo, Oslo, Norway \\ alinaskorynina@gmail.com
}

\begin{abstract}
This investigation is devoted to metal-organic frameworks (MOFs) with UiO-67 topology, the materials with a three-dimensional porous structure and high surface area. Due to the diversity of species, MOFs are used in such areas as luminescent sensors, catalysts, filters, storage and transportation of light gases, and many others [1]. Using noble metals to functionalize metal-organic frameworks is a promising way for constructing new materials for catalytic applications [2,3]. Although numerous successful synthesis of MOFs functionalized by metal ions and metal nanoparticles were reported, the exact mechanisms of structural evolution of the metal sites in many cases are still unknown. Determination of these mechanisms as well as investigation of the intermediate active sites formed during the synthesis is important for tailoring the specific catalytic properties of materials. In this work, we investigate structural changes in UiO-67 functionalized by $\mathrm{Pd}$ and Pt depending on the activation conditions by a combination of theoretical and experimental techniques.
\end{abstract}

Functionalization of UiO-67 by Pd and Pt was achieved via substitution of $10 \%$ standard biphenyl dicarboxylate linkers by $\mathrm{MCl}_{2}-2,2-$ bipyridine-5,5-dicarboxylic acid $\left(\mathrm{MCl}_{2}\right.$ bpydc, $\mathrm{M}=\mathrm{Pd}$, $\mathrm{Pt}$ ) [4, 5]. The obtained materials were further activated by heating to $300{ }^{\circ} \mathrm{C}$ in inert $(\mathrm{He})$ and reducing $\left(\mathrm{H}_{2} / \mathrm{He}\right)$ atmospheres. Evolution of the atomic and electronic structure was monitored by in situ extended Xray absorption fine structure (EXAFS), X-ray absorption near edge structure (XANES) spectroscopies and X-ray powder diffraction (XRPD). All spectroscopic data for Pd $K$ - and Pt $L_{3}$-edges were analysed simultaneously by MCR-ALS approach [6] to determine the number of pure species formed during the activation and their spectra.

To interpret the experimental data, we have performed DFT-calculations and XANES simulation by FDMNES code for different potential intermediates. The atomic models included the initial $\mathrm{MCl}_{2}$ bpydc linker and a number of possible reaction pathways in presence of $\mathrm{H}_{2}$ substitution of both chlorine atoms by hydrogen atoms with formation of $\mathrm{Cl}_{2}$ molecule, substitution of one chlorine by hydrogen atom with formation of $\mathrm{HCl}$ molecule; detachment of one or two chlorines with formation of $\mathrm{HCl}$ molecules, detachment of $\mathrm{MCl}_{2}$ fragment from the linker with its substitution by two hydrogens bonded to nitrogen atoms of the linker; and simulating inert conditions: simple detachment of $\mathrm{MCl}_{2}$ fragment, detachment of chlorines with formation of $\mathrm{Cl}_{2}$ molecule. All reaction pathways were ranged according to the calculated reaction enthalpies and XANES spectra were calculated for the most probable ones.

The reaction pathways with the lowest reaction enthalpies were verified by good agreement between calculated and experimentally observed XANES spectra. For UiO-67-Pd, detachment of $\mathrm{PdCl}_{2}$ is the most probable pathway in both inert and $\mathrm{H}_{2}$ atmospheres which correlate with experimental results. For UiO-67-Pt, four different structures have been identified. In the presence of hydrogen, detachment of one chlorine atom should occur first. The second possible transition in the same environment is the detachment of $\mathrm{PtCl}_{2}$ from the linker with the addition of two hydrogen atoms to nitrogen atoms with the further formation of $\mathrm{Pt}$ nanoparticles at temperatures above $200{ }^{\circ} \mathrm{C}$. While formation of bare Pt-sites occurs from 200 to $300{ }^{\circ} \mathrm{C}$ in the inert flow [7, 8]. Thus, the XANES spectroscopy supported by theoretical calculations allowed verifying and describing intermediate states from the experimental spectra.

[1] Evans J. D., et al., Coord Chem Rev. (2019) 380 378-418.

[2] Tanabe K. K., Cohen S. M., Chem Soc Rev. (2011) 40 (2) 498-519.

[3] Wang Z., Cohen S. M., Chem Soc Rev. (2009) 38 (5) 1315-29.

[4] Braglia L., et al., Phys Chem Chem Phys. (2017) 19 (40) 27489-27507.

[5] Bugaev A. L., et al., Faraday Discuss. (2018) 208 287-306.

[6] Jaumot J., et al., Chemom Intell Lab Syst. 140 (2015) 1-12.

[7] Bugaev A. L., Skorynina A.A., et al., Catal. Today. (2019), 336, 33-39.

[8] Bugaev A. L., Skorynina A.A., et al., Data Brief. (2019) 25, 104208.

Keywords: XANES; XRD; MOFs; MCR; DFT

This research was supported by the Russian Science Foundation, project № 20-43-01015. 